\title{
FIBRATIONS OVER A CWh-BASE
}

\author{
ROLF SCHÖN
}

\begin{abstract}
This note provides a short argument for the known fact that the total space of a fibration has the homotopy type of a CW-complex if base and fiber have.
\end{abstract}

1. Notation. $F \rightarrow E \rightarrow B$ is a (Hurewicz) fibration. A CWh-space is a space having the homotopy type of a $\mathrm{CW}$-complex. The following result is due to Stasheff [9, Proposition (0)]. ${ }^{1}$

2. Theorem. $E$ is a $C W h$-space if $F$ and $B$ are.

Proof. We replace the inductive construction of [9] by the CW approximation theorem [8, p. 412] that is due to Whitehead [11]: to the topological space $E$ there exists a $C W$-complex $X$, called ' $C W$-substitute for $E$ ' in $[10$, p. 97], and a weak homotopy equivalence $f: X \rightarrow E$. We make $f$ into a fibration by taking the associated mapping path fibration $q: P_{f} \rightarrow E$, see e.g. [8, p. 99]. Then $q$ is a weak homotopy equivalence too, and $P_{f}$ is a CWh-space. Therefore $p q$ is a fibration with a CWh-fiber by 3 below. Hence $q$ induces a genuine homotopy equivalence between the fibers of $p q$ and $p$ and is therefore a fiber homotopy equivalence by $[3,6.3]$.

3. Proposition. $F$ is a $C W h$-space if $E$ and $B$ are.

Proof. Compare [9, Corollary (13)]. By coglueing homotopy equivalences, see $[4,(1.2)]$ or $[5,(8.7)]$, the pullbacks of the horizontal rows in the following diagram are homotopy equivalent.

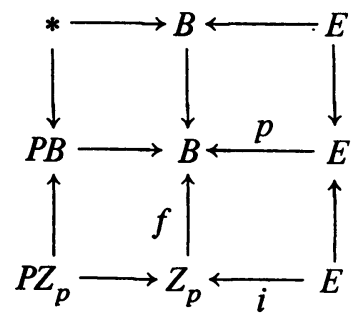

$f i$ is the standard factorization of $p$ over its mapping cylinder $Z_{p}, P Z_{p} \rightarrow Z_{p}$, $P B \rightarrow B$ are the fibrations of paths starting from a point $b \in Z_{p}$, resp.

Received by the editors March 8, 1976 and, in revised form, July 19, 1976.

AMS (MOS) subject classifications (1970). Primary 55F05, 54E60.

${ }^{1}$ As it is remarked in [7, p. 27] Stasheff's proof is not correct, but can be patched.

(C) American Mathematical Society 1977 
$f(b)=* \in B$, and the other arrows are obvious. The upper pullback is the fiber $F$ (over *), the lower one is the space of the paths on the CWh-space $Z_{p}$ starting from $b$ and ending in $E \subset Z_{p}$, and is therefore a CWh-space by [6].

4. Remark. If we assume that $F$ and $E$ are $C W h$-spaces, then the following is true: (a) $B$ is not a CWh-space in general. Fiber and total spaces of Example 2.4 .8 of $[8$, p. 77$]$ are contractible, but the base space, the "Warsaw circle", is not contractible, because it has the nonvanishing Cech homotopy group $\check{\pi}_{1}(B) \cong \mathbf{Z}[2, \S 6]$. (b) the loop space $\Omega B$ is a CWh-space, because it is homotopy equivalent to the fiber of the inclusion $F \rightarrow E[10,2.56]$, and by delooping homotopy equivalences, see [1], $B$ is a CWh-space too, if it is pathconnected and has a numerable, null homotopic covering.

\section{REFERENCES}

1. G. Allaud, De-looping homotopy equivalences, Arch. Math. (Basel) 23 (1972), 167-169. MR 46 \#8217.

2. R. Ciampi and G. De Cecco, Gruppi d'omotopia di Čech, An. Univ. Bucureşti Mat.-Mec. 22 (1973), no. 2, 87-101. MR 50 \# 14739.

3. A. Dold, Partitions of unity in the theory of fibrations, Ann. of Math. (2) 78 (1963), 223-255. MR 27 \#5264.

4. R. Brown and P. R. Heath, Coglueing homotopy equivalences, Math. Z. 113 (1970), 313-325. MR 42 \#1120.

5. K. H. Kamps, Kan-Bedingungen und abstrakte Homotopietheorie, Math. Z. 124 (1972), 215-236. MR 45 \#4412.

6. J. W. Milnor, On spaces having the homotopy type of a CW-complex, Trans. Amer. Math. Soc. 90 (1959), 272-280. MR 20 \#6700.

7. J. P. May, Classifying spaces and fibrations, Mem. Amer. Math. Soc. 1 (1975), issue 1, no. 155. MR 51 \#6806.

8. E. H. Spanier, Algebraic topology, McGraw-Hill, New York, 1966. MR 35 \# 1007.

9. J. D. Stasheff, A classification theorem for fibre spaces, Topology 2 (1963), 239-246. MR 27 \#4235.

10. R. M. Switzer, Algebraic topology-homotopy and homology, Springer-Verlag, New York, 1975. 43.

11. J. H. C. Whitehead, A certain exact sequence, Ann. of Math. (2) 52 (1950), 51-110. MR 12,

Mathematisches Institut der Universität, 69 Heidelberg 1, im Neuenheimer feld 288, Federal Republic of Germany 